Revue de l'Institut des langues et cultures

d'Europe, Amérique, Afrique, Asie et Australie

$21 \mid 2015$

Discours politique et culturel dans la Russie

contemporaine

\title{
Le rôle des soviétismes dans les stratégies du discours politique russe contemporain
}

The Role of Sovietisms in the Strategies of Contemporary Russian Political Discourse

Valéry Kossov

\section{OpenEdition}

Journals

Édition électronique

URL : http://journals.openedition.org/ilcea/3045

DOI : 10.4000/ilcea.3045

ISSN : 2101-0609

Éditeur

UGA Éditions/Université Grenoble Alpes

Édition imprimée

ISBN : 978-2-84310-294-3

ISSN : $1639-6073$

Référence électronique

Valéry Kossov, «Le rôle des soviétismes dans les stratégies du discours politique russe

contemporain », ILCEA [En ligne], 21 | 2015, mis en ligne le 01 février 2015, consulté le 01 mai 2019

URL : http://journals.openedition.org/ilcea/3045 ; DOI : 10.4000/ilcea.3045

Ce document a été généré automatiquement le 1 mai 2019.

(C) ILCEA 


\section{Le rôle des soviétismes dans les stratégies du discours politique russe contemporain}

The Role of Sovietisms in the Strategies of Contemporary Russian Political

Discourse

Valéry Kossov

1 Le discours politique russe contemporain a subi de multiples transformations depuis la désagrégation de l'URSS. Cependant, le « soviétique » ne semble pas complètement oublié dans le discours du pouvoir et remplit certaines fonctions dans les stratégies de discours et de communication, qui constitueront l'objet de cet article.

2 L'étude des soviétismes a suscité un intérêt croissant et constant, en Russie, mais aussi en France, depuis l'ouvrage d'André Mazon (1920). Actuellement, les diverses études des soviétismes, qui ont changé de statut depuis Mazon, se font à partir de la presse, dans les sociolectes, dans le discours, ce qui a même fait émerger une nouvelle discipline, la «soviétologie linguistique» (БУДАЕв \& чУдинов, 2009), où les chercheurs se sont efforcés de dégager un système à partir de l'emploi des soviétismes dans la langue, le langage et le discours.

3 Pourtant, des interrogations existent toujours sur la possibilité d'une définition unique du terme "soviétisme ». Les premières occurrences de ce terme se rencontrent dans un ouvrage de Geliy Tchernov (ЧЕРНОВ, 1958:4) où le mot "soviétisme" s'écrit entre guillemets et est associé aux realia n'ayant pas d'équivalent dans les langues étrangères. À partir des années 1960, le terme "soviétisme » est couramment utilisé dans les ouvrages portant sur la théorie de la traduction, par analogie avec les germanismes, les anglicismes, les gallicismes (КАТЦЕР \& КУнинА, $1964: 40$ ).

Dans notre étude, on entend d'une manière large sous le terme "soviétisme » tout mot, locution ou énoncé contribuant à la reconstitution explicite ou implicite du monde et de l'époque soviétique dans le discours politique contemporain. 
Étant donné la largeur du champ sémantique de « discours politique », nous nous sommes limités à un corpus de textes représentatifs du discours du pouvoir en tant que discours institutionnel, c'est-à-dire celui qui incarne le pouvoir et qui émane des détenteurs du pouvoir politique, ou des communicants mandatés par ce pouvoir, pour le représenter en Russie ou à l'étranger. Le discours institutionnel est censé faire passer un message du pouvoir à un certain public cible (électeurs, fonctionnaires, groupes socio-professionnels) et façonner l'image du pouvoir. Il sera donc question du discours du tandem «Président - Premier ministre » et de son entourage proche, qui soit est à l'origine du discours institutionnel, soit constitue le relais des thèses principales exprimées dans ce type de discours (représentants de l'Administration présidentielle, certains conseillers). Le corpus est constitué d'interventions publiques, interviews et conférences de presses, c'est-à-dire de textes se rapportant au genre oral par leur forme et interactif par leur fonction.

6 Ainsi, nous commencerons par une question générale : en quoi le recours aux soviétismes peut-il être considéré comme une stratégie du discours du pouvoir?

\section{Les stratégies du discours}

7 Remarquons tout d'abord que le terme de «stratégie » a pour référent l'art de conduire les opérations militaires dans le but de remporter la victoire. Il est également employé dans plusieurs théories scientifiques, comme la théorie des jeux (Von Neumann \& Morgenstern, $1964: 44$ ), la psychologie cognitive (Esperet, $1990: 8$ ), la psychologie sociale (Chabrol, 1990 : 216). En linguistique et en communication politique, les stratégies du discours sont étudiées à partir de méthodologies diverses. Nous pouvons évoquer les approches des linguistes russes : pragmatiques (ТРУФАНОВА, 2001: 58 ; МАКАРОВ, 2003 : 192), cognitives (ИССЕРС, 1999: 100), psycholinguistiques (ВЕРЕТЕНКИНА, 2001: 178). Compte tenu de cette diversité d'approches, nous pouvons considérer, dans cet article, comme stratégie du discours une orientation particulière du comportement discursif, choisie pour atteindre certains objectifs précis de l'acte de communication.

Dans notre démarche, nous nous appuierons sur un classement général des stratégies en fonction des objectifs de la communication. Patrick Charaudeau distingue trois groupes d'enjeux autour desquels se développent les stratégies du discours. C'est à partir de ces trois groupes que nous nous efforcerons de classer les types de stratégies. Exposons-les brièvement ici.

9 L'enjeu de légitimation ou de position d'autorité du sujet se fonde sur la construction de l'autorité institutionnelle et donne lieu à la stratégie d'autoreprésentation. Cet enjeu se fonde également sur la construction de l'autorité personnelle et s'associe à la stratégie de persuasion, d'argumentation ou d'agitation.

10 L'enjeu de crédibilité ou de position de vérité sert de fondement pour les stratégies informationnelle et interprétative, la stratégie d'émotions, la stratégie argumentative où l'on privilégie les tactiques de distanciation et d'accentuation.

11 L'enjeu de captation, consistant à faire entrer le partenaire dans l'échange communicatif, peut se réaliser sous forme de stratégies de discréditation, d'émotions, d'attaque et de défense, d'agitation et de manipulation (Charaudeau, 1998b : 13-14). l'information de telle sorte que celle-ci s'apparente à un spectacle qui doit toucher la 
sensibilité du spectateur (Charaudeau, 1994b : 17), ce qui donne la production des effets discursifs de connivence (jeux de mots), d'émotion (description du « désordre social») (Charaudeau, $2000: 148$ ).

13 Certes, ce classement, où les stratégies se recoupent en fonction des enjeux, ne prétend pas à l'exhaustivité. Il nous servira de point de départ pour évaluer le rôle et les fonctions des soviétismes dans les stratégies du discours. Cela nous amènera à la question plus générale des objectifs que le pouvoir russe poursuit en adaptant les soviétismes à ses stratégies du discours.

\section{La légitimation du pouvoir à travers les soviétismes}

L'enjeu de légitimation suppose le déploiement de stratégies visant à déterminer la position d'autorité du sujet parlant. Dans notre cas, il s'agit des communicants du pouvoir.

Les stratégies du discours s'appuient sur deux types de construction :

- autorité institutionnelle fondée par le statut du sujet lui conférant autorité de savoir (expert, savant, spécialiste) ou de pouvoir de décision (responsable d'une organisation, chef d'État ou de gouvernement) ;

- autorité personnelle fondée sur l'activité de persuasion et de séduction du sujet qui lui donne une autorité de fait pouvant se superposer à la précédente. (Charaudeau, 1998b : 13)

Quel rôle jouent les soviétismes dans les stratégies de légitimation? Quels types de soviétismes sont propres à ces stratégies?

Dans la stratégie d'autoreprésentation, le discours du pouvoir construit une image institutionnelle ou personnelle de Soi par association, solidarité, ou distanciation, voire opposition vis-à-vis de l'époque soviétique.

Dans le cas des références explicites à l'URSS, la stratégie d'autoreprésentation par distanciation ou opposition se révèle récurrente. Dans ce cas, c'est l'autorité institutionnelle, celle du président ou du premier-ministre, qui est mise en avant :

Я ПОМНЮ БРЕЖНЕВСКИЕ ВРЕМЕНА, ПОМНЮ ВРЕМЕНА АНДРОПОВА, ПОМНЮ ВРЕМЕНА КОНСТАНТИНА УСТИНОВИЧА ЧЕРНЕНКО, ПОМНЮ ВРЕМЕНА МИХАИЛА СЕРГЕЕВИЧА ГОРБАЧЁВА. ЧТО БЫ МНЕ НИ ГОВОРИЛИ - ЭТО ДРУГИЕ ВРЕМЕНА, И СТРАНА У НАС БЫЛА ДРУГОЙ. ХОРОШИЕ, ПЛОХИЕ, АВТОРИТАРНЫЕ ИЛИ ЖЕ ДЕМОКРАТИЧНЫЕ, МЕДВЕДЕВ, ПУТИН, НО МЫ ВСЁ-ТАКИ ИЗ ДРУГОЙ ЭПОХИ. (МЕДВЕДЕВ, 15.10.2011)

"Je me rappelle l'époque de Brejnev, celle d'Andropov, celle de Konstantin Oustinovitch Tchernenko, celle de Mikhail Sergueevitch Gorbatchev. Quoi qu'on en dise, c'était un autre temps, et un autre pays. Qu'on soit bons, mauvais, autoritaires ou bien démocratiques, Medvedev, Poutine, nous sommes quand même d'une autre époque. » (Medvedev, 15 octobre 2011)

Dans l'exemple suivant, il s'agit davantage de distanciation que d'opposition, car l'intention du pouvoir n'est plus de critiquer l'époque soviétique, mais d'accentuer l'information positive relative à l'action politique actuelle, qui se veut nettement distincte de l'action du pouvoir soviétique :

ВО-ПЕРВЫХ, И В СОВЕТСКИЕ ВРЕМЕНА, И ДАЖЕ В НАЧАЛЕ 1990-Х - Я НЕ ХОЧУ, ЧТОБЫ ЭТО ВЫГЛЯДЕЛО, КАК ОГУЛЬНАЯ КРИТИКА - БЫЛО МНОГО И ПОЗИТИВНОГО, НО Я ЧТО-ТО НЕ ПРИПОМНЮ, ЧТОБЫ ПОСЛЕВОЕННОЕ СОВЕТСКОЕ РУКОВОДСТВО, ЛИДЕРЫ СОВЕТСКИЕ ПОСЛЕВОЕННЫЕ ТАК ЖЕ ИНТЕНСИВНО РАБОТАЛИ, КАК ЭТО ДЕЛАЮ Я ИЛИ ДЕЙСТВУЮЩИЙ ПРЕЗИДЕНТ МЕДВЕДЕВ ДМИТРИЙ АНАТОЛЬЕВИЧ. (ПУТИН, 17.10.2011) 
«D'abord, à l'époque soviétique, et même au début des années 1990, et je ne voudrais pas qu'on voit ici une critique débridée, il y avait aussi beaucoup de choses positives, mais autant que je m'en souvienne, les dirigeants soviétiques d'aprèsguerre, les leaders soviétiques d'après-guerre travaillaient avec autant d'intensité que moi aujourd'hui, ou que le président en exercice Dmitri Anatolevitch Medvedev. » (Poutine, 17 octobre 2011)

C'est plus largement l'évocation du modèle économique socialiste (comme ayant mené le pays au désastre de 1991), qui permet au pouvoir de recourir à la tactique de distanciation afin de mettre en valeur son image et sa légitimité :

\begin{abstract}
ТО, ЧТО МЫ ДЕЛАЕМ, УБЕЖДАЕТ МЕНЯ, ЧТО МЫ НА ПРАВИЛЬНОМ ПУТИ. КОНЕЧНО, МЫ НЕ МОГЛИ НЕ УЧИТЫВАТЬ РЕАЛИИ. ВСЯ ФИНАНСОВАЯ СИСТЕМА РАЗВАЛИЛАСЬ ПРИ СССР, ВСЯ СОЦИАЛЬНАЯ, У НАС ЭКОНОМИКА НАЧАЛА РУШИТЬСЯ, ПОТОМУ ЧТО БЫЛА НАСТРОЕНА НА ЗАКРЫТОЕ ПРОИЗВОДСТВО [...] ЖЕЛЕЗНЫЙ ЗАНАВЕС, КОГДА ПОТРЕБЛЯЕТСЯ ТОЛЬКО ТО, ЧТО ПРОИЗВОДИТСЯ, ПРИЧЕМ ПОТРЕБЛЯЕТСЯ ЛЮБОГО КАЧЕСТВА. (ПУТИН, in КОЛЕСНИКОВ, 30.08.2010)

«Ce que nous faisons me conforte dans l'idée que nous sommes sur la bonne voie. Bien sûr, nous avons dû tenir compte des réalités. Tout le système financier s'est effondré à l'époque de l'URSS, tout le système social, notre économie a commencé à se détruire, parce qu'elle était conçue pour une production fermée. [...] Le rideau de fer, c'est lorsqu'on ne consomme que ce que l'on produit, et on le consomme quelle qu'en soit la qualité. » (Poutine, 30 août 2010)
\end{abstract}

Dans ses stratégies d'autoreprésentation par références explicites au soviétique, le pouvoir emploie principalement le procédé de distanciation. Cela semble faire partie de la rhétorique générale, qui ne laisse aucun doute sur le fait que le retour à l'URSS n'est pas envisageable pour le pouvoir, ce que confirme sans équivoque Vladimir Poutine :

И МЫ ЖЕ НЕ ГОВОРИМ О КАКОМ-ТО ПОЛИТИЧЕСКОМ ОБЪЕДИНЕНИИ, О ВОЗРОЖДЕНИИ СОВЕТСКОГО СОЮЗА, ДА РОССИЯ ДАЖЕ И НЕ ЗАИНТЕРЕСОВАНА В ЭТОМ СЕГОДНЯ. (ПУТИН, 17.10.2011)

«Et nous ne parlons même pas d'une union politique, ni d'une renaissance de l'Union soviétique, la Russie n'est même pas intéressée par cela aujourd'hui.»

(Poutine, 17 octobre 2011)

Dans cet exemple l'association, dans l'autoreprésentation, se fait entre le "nous » collectif et la Russie. Nous n'avons pas trouvé d'exemples où le pouvoir s'identifierait nettement à l'époque soviétique par des références explicites. En revanche, dans le champ de l'implicite, nous avons constaté l'utilisation des autres procédés de la stratégie d'autoreprésentation, particulièrement en ce qui concerne la construction de l'image personnelle.

\title{
L'évocation implicite des soviétismes dans la stratégie d'autoreprésentation
}

22 En effet, le lexique de l'époque soviétique est souvent repris tel quel dans le discours du pouvoir, avec ou sans changement sémantique, sans toutefois que soient perceptibles la prise de conscience ou la mise en valeur de la connotation soviétique. Il s'agit donc d'un emploi inconscient ou non intentionnel de mots autrefois courants dans le discours politique, tels que graždanin, otečestvo, rodina, tovarišč, klass.

23 Ainsi, ce phénomène peut prendre la forme d'un réemploi des mots relevant de la composante patriotique de l'idéologie soviétique. Comme nous le montre l'exemple suivant, il s'agit du procédé de manifestation de la solidarité des leaders politiques avec la 
société, ou de l'État avec son peuple, dans le cadre de l'autoreprésentation personnelle du pouvoir :

ПОДЧЁРКИВАЮ, И ДЛЯ МЕНЯ, И ДЛЯ ВЛАДИМИРА ПУТИНА ГЛАВНЫМ ЯВЛЯЕТСЯ ПРИНОСИТЬ ПОЛЬЗУ НАШЕМУ ОТЕЧЕСТВУ, НАШЕМУ ГОСУДАРСТВУ. (МЕДВЕДЕВ, 30.09.2011)

« Je le souligne, pour moi comme pour Vladimir Poutine, l'essentiel est d'être utile à notre patrie, à notre État. » (Medvedev, 30 septembre 2011)

ВОТ ЭТО, Я СЧИТАЮ, КОНСТИТУЦИОННЫЙ ДОЛГ, ЕСЛИ ХОТИТЕ, ЛЮБОГО ГРАЖДАНИНА, КОТОРОМУ НЕБЕЗРАЗЛИЧНА СУДЬБА СОБСТВЕННОЙ СТРАНЫ, СВОЕЙ РОДИНЫ. (МЕДВЕДЕВ, 30.09.2011)

«Cela, si vous voulez, j'estime que c'est le devoir constitutionnel de tout citoyen qui n'est pas indifférent au destin de son propre pays, de sa patrie.» (Medvedev, 30 septembre 2011)

La stratégie d'autoreprésentation par solidarité peut également se présenter sous un angle nouveau. Comme on le voit dans l'exemple suivant, le mot klass est associé, suite à un glissement sémantique volontaire, à un corps de métiers, et l'intention du sujet parlant consiste à réhabiliter les fonctionnaires, sans pourtant les associer au système de la fonction publique soviétique :

МОЖНО ЧТО УГОДНО ТАМ ГОВОРИТЬ: КОРРУПЦИЯ, ВЗЯТКИ. НО ЭТО ОСКОРБЛЯЕТ ЦЕЛЫЙ КЛАСС ОЧЕНЬ УМНЫХ, ВЫСОКОГО КАЧЕСТВА ЛЮДЕЙ. (СУРКОВ, 15.02.2010)

«On peut dire ce qu'on veut: corruption, pots-de-vin. Mais c'est une offense pour toute une classe de gens très brillants, de première catégorie. » (Sourkov, 15 février 2010)

L'emploi explicite des soviétismes peut se transformer en implicite, toujours dans le cadre de la stratégie d'autoreprésentation, lorsque le soviétisme est censé véhiculer des émotions. Il s'agit, dans l'exemple suivant, d'une utilisation ironique du mot tovarišč. Cette ironie, intentionnelle, participe bien à la construction d'une autorité personnelle, respectueuse, mais aussi dominatrice :

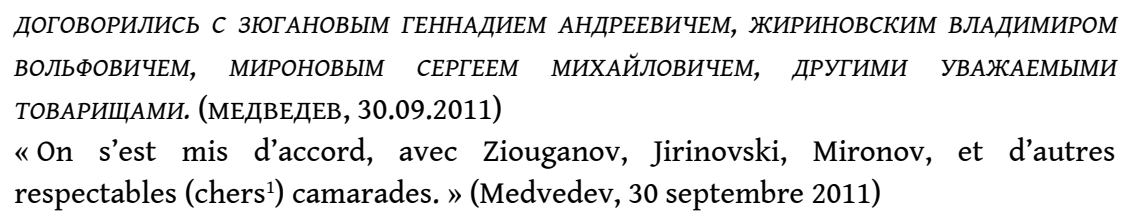

作 fréquent lorsqu'il s'agit de mots de référence implicite. Dans la théorie de l'interdiscursivité, ce phénomène langagier consiste à utiliser le mot comme un repère, un marqueur, qui renvoie non pas directement au référent, mais à un certain nombre de traits distinctifs du mot.

Dans le discours du pouvoir actuel, le nombre de références implicites est élevé. Elles se rapportent aux realia soviétiques relatifs soit aux domaines politique et social, soit au domaine culturel.

Par exemple, lorsqu'il donne sa "parole d'honneur", Vladimir Poutine se réfère explicitement à son appartenance au parti "Russie unie ", mais l'expression employée renvoie ses interlocuteurs au passé soviétique, où la parole d'honneur constituait un cliché utilisé à tous les niveaux de l'organisation politique : čestnoe oktjabrjatskoe, čestnoe pionerskoe, čestnoe kosomol'skoe :

ПОВЕРЬТЕ МНЕ: Я ЭТОГО НЕ ЗНАЮ! Я ЭТИМ НЕ ЗАНИМАЮСЬ! Я ГОВОРЮ ОТКРОВЕННО И ДАЮ ВАМ ЧЕСТНОЕ ПАРТИЙНОЕ СЛОВО! (ПУТИН, 30.08.2010) 
"Je vous prie de me croire, je ne sais pas cela. Je ne m'occupe pas de cela. Je vous le dis en toute sincérité et je vous en donne ma parole sincère de membre du Parti! » (Poutine, 30 août 2010) particuliers (subbotnik, voskresnik), pour parler des travaux de nettoyage et de la création d'un parc national sur la terre François-Joseph en Arctique. Cette référence s'avère suffisamment fonctionnelle et expressive pour mettre l'accent sur les volumes importants de travail à faire :

И ЧТО НАМ НУЖНО СЕЙЧАС СДЕЛАТЬ - БЕЗУСЛОВНО, ОРГАНИЗОВАТЬ ГЕНЕРАЛЬНУЮ УБОРКУ В АРКТИКЕ. НЕ ТОЛЬКО СУББОТНИК, НО И ВОСКРЕСНИК. И ПО ДРУГИМ ДНЯМ ПРИДЕТСЯ ПОРАБОТАТЬ. (ПУТИН іn СУББОТИНА, 16.10.2012)

«Et ce que nous devons faire à présent, c'est incontestablement organiser un grand ménage en Arctique. Pas juste un samedi rouge, mais aussi un dimanche, et tous les autres jours il faudra aussi travailler. » (Poutine, 16 octobre 2012)

Cet effet de connivence avec les interlocuteurs du pouvoir se renforce encore lorsqu'il s'agit de références aux réalités culturelles soviétiques, qui sont souvent véhiculées par des citations tirées de films cultes de l'époque. Elles peuvent s'employer dans le but de donner une appréciation négative ou positive. C'est, par exemple, le cas de l'expression « vor dolžen sidet' v tjur'me» («la place du voleur est en prison»), utilisée à propos de la libération de Mikhaill Khodorkovski (Poutine, 16 décembre 2010) qui est une réplique du personnage principal du film de Stanislav Govoroukhine Mesto vstreči izmenit' nel'zja.

31 Toutefois, parmi les références culturelles, les marqueurs à connotations positives sont plus fréquents, et ils remplissent une fonction d'animation du discours, mais aussi celle d'identification commune dans la référence entre le sujet parlant et ses interlocuteurs. Dans l'exemple suivant, Vladimir Poutine, expliquant son point de vue sur les rapports économiques entre la Russie et l'Union européenne, introduit à la fin une référence au film culte soviétique de 1978 D'Artan'jan i tri mušketera :

\begin{abstract}
А ПРОБЛЕМА В ТОМ, ЧТО ВСЕ ЭТИ СТРАНЫ В СВОЁ ВРЕМЯ БЫЛИ ПРИНЯТЫ В ЕВРОСОЮЗ, И ЕВРОСОЮЗ ВЗЯЛ НА СЕБЯ ВСЕ ОБЯЗАТЕЛЬСТВА ПО СУБСИДИРОВАНИЮ ИХ ЭКОНОМИК. [...] ТО ЕСТЬ ОБЪЕДИНЁННАЯ ЕВРОПА ХОЧЕТ СОХРАНИТЬ ПОЛИТИЧЕСКОЕ ВЛИЯНИЕ И ЧТОБЫ МЫ ЗА ЭТО ЕЩЁ НЕМНОЖКО ЗАПЛАТИЛИ. НО ЭТО НЕКОНСТРУКТИВНЫЙ ПОДХОД. ЭТО ПЕРВАЯ ЧАСТЬ МАРЛЕЗОНСКОГО БАЛЕТА. (ПУТИН, 09.09.2012)

« Le problème, c'est que tous ces pays, à un moment ou à un autre, ont été acceptés dans l'Union européenne, et l'Europe s'est engagée à garantir par ses subventions leurs économies. [...] C'est à dire que l'Union européenne veut conserver son influence politique, et nous faire payer encore un peu plus. Mais ce n'est pas une approche constructive. C'est la première partie du ballet de la Merlaison.» (Poutine, 9 septembre 2012)
\end{abstract}

Le ballet de la Merlaison évoqué ici par Poutine est un épisode du film qui se déroule dans une ambiance calme et solennelle, avec un maître de cérémonie qui en annonce les parties successives. La première partie symbolise un calme cérémonieux tout relatif, car au même moment d'Artagnan est en train de franchir de multiples obstacles, afin de faire parvenir les ferrets à la reine. Au moment de l'annonce de la deuxième partie du ballet, dans le film, il tombe sur le maître de cérémonie du palais, et le ballet est arrêté par une suite d'actions désordonnées, ce qui donne à l'expression « la deuxième partie du ballet de Merlaison » le sens métaphorique d'un véritable chaos. Même si le public russe n'a pas en mémoire les détails du film, cette citation est assez facilement identifiable, et elle permet au locuteur d'aménager une pause humoristique dans un propos au sujet sérieux. 

d'innovation de Skolkovo, Dmitri Medvedev fait référence à une phrase du film d'Eldar Riazanov Beregis' avtomobilja qui soulève les rires dans la salle, du fait de son caractère reconnaissable et du contraste contextuel par rapport à Skolkovo (vu que le personnage du film parle de la Constitution soviétique) :

ПОЭТОМУ КОГДА «СКОЛКОВО» КРИТИКУЮТ СРЕДСТВА МАССОВОЙ ИНФОРМАЦИИ, КОНЕЧНО, ОНИ ПОДНИМАЮТ РУКУ НА САМОЕ СВЯТОЕ, ЧТО У НАС ЕСТЬ. (МЕДВЕДЕВ, 15.10.2011)

«Donc, quand ils critiquent Skolkovo, les médias lèvent la main sur ce que nous avons de plus sacré. » (Medvedev, 15 octobre 2011)

La fonction principale de l'emploi des mots de référence implicite consiste, pour le pouvoir, à se représenter en phase avec la population, qui pour une grande part s'identifie et se reconnaît dans ces allusions aux réalités soviétiques. Dans le cadre de la stratégie de l'autoreprésentation personnelle, ce procédé d'identification par l'implicite demeure un moyen assez efficace de mise en valeur de sa propre légitimité.

Tout comme les références implicites, les métaphores ou expressions métaphoriques se référant au monde soviétique occupent une place importante dans la stratégie d'autoreprésentation du pouvoir. Il peut s'agir d'une métaphore empruntée directement au discours soviétique, avec ou sans reprise du sens initial. Ainsi, les expressions « kuznica arktičeskix kadrov ", « ruki po lokot' v krovi », « podstavit' plečo », « protjanut' ruku pomošči », sont employées dans leur sens initial.

(ans lexemple suivant, la métaphore du "gendarme» n'est pas d'origine soviétique, mais elle renvoie au modèle de la « force injuste », récurrent dans le discours soviétique. Toutefois, le procédé d'opposition implicite, voire euphémistique à l'Autre, ici les ÉtatsUnis, s'inscrit dans la stratégie d'autoreprésentation qui était propre au discours soviétique, avec le «nous » concret et le "quelqu'un », en apparence indéterminé, mais indirectement connu de tous, étant donné l'emploi récurrent depuis les années 1990 de cette tournure pour désigner les États-Unis :

НО КОРЧИТЬ ИЗ СЕБЯ МИРОВОГО ЖАНДАРМА НАМ НИ К ЧЕМУ, ЕСЛИ ЭТО КОМУ-ТО НРАВИТСЯ, ПУСТЬ ЭТИМ ЗАНИМАЕТСЯ КТО-ТО ДРУГОЙ. (ПУТИН, 17.10.2011)

«Mais nous n'avons pas envie de nous faire passer pour le gendarme du monde, si cela plait à quelqu'un d'autre, qu'il le fasse. » (Poutine, 17 octobre 2011)

La même tactique de distanciation est employée lorsque l'Autre est désigné par un euphémisme provenant de la langue des affaires, le mot "concurrent». Cette fois l'allusion semble renvoyer d'une manière plus générale à tous les pays développés occidentaux :

ГЛАВНАЯ БОРЬБА ИДЁТ ЗА МИРОВОЕ ЛИДЕРСТВО, И ЗДЕСЬ МЫ С КИТАЕМ СПОРИТЬ НЕ СОБИРАЕМСЯ. ЗДЕСЬ У КИТАЯ ДРУГИЕ КОНКУРЕНТЫ. ВОТ ПУСТЬ ОНИ МЕЖДУ СОБОЙ И РАЗБИРАЮТСЯ. (ПУТИН, 17.10.2011)

«La lutte principale, c'est pour la place de leader mondial, et nous n'avons pas l'intention de la disputer à la Chine. Elle a d'autres concurrents. Ils n'ont qu'à régler ça entre eux. » (Poutine, 17 octobre 2011)

Certains euphémismes, utilisés dans les tactiques de distanciation, sont formés à partir de la combinaison de modèles d'expressions politiquement correctes occidentales et soviétiques. Ainsi, l'expression «prinuždenie k miru» («la paix par la contrainte»), apparue dans le discours à la suite de la guerre en Géorgie de 2008, reprend à la fois certains traits de "la lutte pour la paix» datant de l'époque soviétique, et des « opérations de pacification » plus récentes se rapportant à la guerre en Yougoslavie et à d'autres conflits armés plus contemporains. Faisant preuve d'un certain sens de l'humour,

ILCEA, 21 | 2015 
Dmitri Medvedev étend ce cliché au domaine des innovations : «Èto voobšče naš metod: prinuždenie togo ili inogo gosudarstva k miru, prinuždenie k inovacijam. » (« En principe, c'est notre méthode : la contrainte d'un tel État à la paix, la contrainte aux innovations.» Medvedev, 15 octobre 2011)

Enfin, l'autoreprésentation s'inscrit également dans la stratégie de persuasion où Vladimir Poutine s'appuie sur la tactique de la promesse, adressée au destinataire extérieur en ce qui concerne le fond, et au destinataire interne quant à la forme. En effet, la métaphore employée ci-dessous appartient au modèle corporel, assez productif dans le discours soviétique :

ЭТОТ ПРОЕКТ ИМЕЕТ ШАНСЫ ПРЕВРАТИТЬСЯ В ОЧЕНЬ БОЛЬШОЙ, БОЛЬШОЙ ПО МЕЖДУНАРОДНЫМ МИРОВЫМ МЕРКАМ, ПОТОМУ ЧТО ЯПОНИЯ ОЧЕНЬ КРУПНЫЙ ПОТРЕБИТЕЛЬ ЭНЕРГОРЕСУРСОВ - СВОИХ ЭНЕРГОРЕСУРСОВ НЕТ, НО МЫ ГОТОВЫ ЗДЕСЬ ПОДСТАВИТЬ ПЛЕЧО ЯПОНЦАМ. (ПУТИН, 09.09.2012)

"Ce projet a toutes les chances de devenir un très grand projet, de dimension internationale, parce que le Japon est un grand consommateur de ressources énergétiques, or il n'a pas de ressources propres, mais nous sommes prêts à offrir notre épaule aux Japonais. » (Poutine, 9 septembre 2012)

La construction de l'image personnelle passe également par le champ lexical où, à l'instar des leaders soviétiques, le pouvoir actuel a recours aux mots et expressions populaires ou familiers.

Cette façon de se vouloir proche du peuple est récurrente et permet d'animer le style officiel, lors des interventions publiques ou des interviews. L'emploi du lexique populaire ou grossier, trait particulier du discours soviétique informel, vise également à se montrer plus expressif, direct, voire provocateur, comme c'est le cas dans une phrase de Vladimir Poutine concernant ses rapports avec le président biélorusse Alexandre Loukachenko : " Pytalsja kak-to capat', no ja kak-to na èto ne zatočilsja. » («Il a essayé de me rentrer dedans, mais ça ne me fait ni chaud ni froid. » Poutine, 30 août 2010)

41 Dans le discours de Vladimir Poutine, la tactique d'association avec le peuple russe se construit souvent par une référence directe aux proverbes, dictons ou mots du langage populaire. Cela s'inscrit dans les stratégies de construction de l'image de chef charismatique qui, dans le cas de Poutine, se traduit parfois par l'adoption des différents comportements des hommes politiques du passé. Notamment, l'emploi fréquent des proverbes dans le passé a été le propre du discours du général Lebed: " "Speška nužna tol'ko pri lovle" - tak u nas v narode govorjat » ("Il n'y a que pour attraper les puces qu'il faut se presser - c'est comme cela qu'on dit dans le peuple chez nous", Poutine, 17 octobre 2011) ; « Kto pokupaet, xotjat polučit' ego podeševle, a lučše voob̌šce na xaljavu, kak u nas $v$ narode govorjat, za besplatno" ("Ceux qui achètent veulent l'avoir moins cher, et mieux encore gratis, comme on dit dans le peuple chez nous, gratuitement », Poutine, 17 octobre 2011).

Les expressions populaires sont beaucoup moins fréquentes dans le discours de Dmitri Medvedev, ce qui semble cohérent avec son image d'homme politique proche de l'intelligentsia, mais aussi avec son statut de second du pouvoir, dont le charisme populaire ne doit pas surpasser celui du maître. Toutefois, les proverbes sont également employés par Dmitri Medvedev dans le cadre de la tactique d'association, ce qui s'apparente à une imitation du discours de Vladimir Poutine.

Ainsi, afin d'asseoir sa légitimité, le pouvoir instrumentalise les soviétismes dans les représentations de Soi et de l'Autre en s'appuyant principalement sur les tactiques 
d'association avec le peuple. Il s'agit de l'usage des moyens implicites comme les métaphores, les euphémismes et allusions, les vulgarismes. Sur le champ des références explicites à l'époque soviétique, c'est au contraire une tactique de distanciation, voire d'opposition qui est déployée, ce qui a pour objectif de dégager l'image positive et crédible du pouvoir actuel.

L'enjeu de crédibilité fait également partie des stratégies de discours du pouvoir russe, et nous nous interrogerons donc sur le rôle des soviétismes dans un discours qui cherche à se rendre crédible.

\section{L'enjeu de crédibilité}

L'enjeu de crédibilité dans les stratégies du discours politique consiste à déterminer une position de vérité, de sorte qu'il puisse être pris au sérieux. Patrick Charaudeau distingue trois types de positionnements :

- se mettre dans une position énonciative de neutralité, effacer dans son mode d'argumentation toute trace de jugement et d'évaluation personnelle ;

- se mettre dans une position d'engagement avec une prise de position dans le choix des arguments et une modalisation évaluative de son discours, ce qui produira un discours de conviction destiné à être partagé par l'interlocuteur ;

- se mettre dans une position de distanciation qui le conduira à prendre l'attitude froide du spécialiste ou expert. (Charaudeau, 1998a : 14)

À première vue, les évocations récurrentes de l'URSS dans le discours du pouvoir actuel apparaissent souvent associées au sentiment de nostalgie et de regret de la désagrégation rapide et irréversible d'une grande puissance. Nous pouvons donner, à titre d'exemple, le propos de Vladimir Poutine qui a été le plus médiatisé en Russie et à l'étranger, et qui lui a donné une image d'un nostalgique de l'URSS ou, du moins, d'un conservateur: «Kto ne žaleet o raspade SSSR, $u$ togo net serdca. A $u$ tex, kto želaet ego vosstanovlenia $v$ prežnem vide, $u$ togo net golovy." ("Qui ne regrette pas la chute de l'URSS n'a pas de cœur. Qui souhaite son rétablissement à l'identique n'a pas de tête. » Poutine, 16 décembre 2010)

Certes, ce propos peut être interprété de multiples façons, concernant sa composante nostalgique. Il s'agit, d'une part, de la stratégie d'appel aux émotions. D'autre part, nous pouvons y constater une prise de position assez nuancée qui s'exprime par les métaphores de "sentiments » et de "raison ». Enfin, le président russe véhicule un message implicite sur sa propre " position de vérité » où la raison n'a pas à tomber sous l'emprise des sentiments. Au final, la stratégie d'appel aux émotions et la figure rhétorique d'une « fausse " alternative entre la « tête » et le "cœur » amène à constituer un ethos du chef de l'État suffisamment crédible pour la majorité des interlocuteurs, nostalgiques ou pas de l'époque soviétique.

Quoi qu'il en soit, les sentiments de regret et de honte pour ceux qui ont échoué à sauvegarder l'intégrité de l'empire soviétique se manifestent de façon récurrente dans le discours du pouvoir. La stratégie d'appel aux émotions peut toutefois s'appliquer de telle sorte que la personnalité du sujet parlant se fonde dans le propos à forte composition doxique. Comme nous le montre l'exemple suivant, Vladimir Poutine tente de s'effacer dans ce jugement, en s'associant au ressenti de la majorité des Russes :

И ЭТОЙ ГОРДОСТИ НАШЕЙ С ВАМИ НЕ ХВАТИЛО ДЛЯ ТОГО, ЧТОБЫ СОХРАНИТЬ ЕДИНУЮ

СТРАНУ. ПОТОМУ ЧТО У ЛЮДЕЙ, ПОДАВЛЯЮЩЕГО БОЛЬШИНСТВА, ВО ВСЯКОМ СЛУЧАЕ, ВОЗНИКЛО ТАКОЕ ОЩУЩЕНИЕ, ЧТО ХУЖЕ НЕ БУДЕТ. (ПУТИН, 17.11.2011) 
«Et cette fierté nous a manqué à nous tous pour conserver un pays uni, parce que les gens, la majorité en tout cas, ont eu l'impression que ça ne pourrait pas être pire. » (Poutine, 17 novembre 2011) commentaire qui semble récurrente dans l'établissement de la position de vérité par rapport à l'époque soviétique. Notamment, le communicant se met dans la posture d'expert qui suppose un discours au caractère analytique et explicite :

РАСПАД СОВЕТСКОГО СОЮЗА, КАК ВЫ ПОМНИТЕ, ПРОИЗОШЕЛ, ПО-МОЕМУ, В ДЕКАБРЕ... У НАС ДВА ТЯЖЕЛЫХ ПЕРИОДА БЫЛО: ЦАРЬ УШЕЛ, И СРАЗУ НАЧАЛИСЬ УЖАСНЫЕ СОБЫТИЯ, И 91-Й ГОД. (ПУТИН, 17.11.2011)

La chute de l'Union soviétique, vous vous en souvenez, s'est produite, je crois, en décembre... Nous avons eu deux périodes dures : quand le tsar est parti, et qu'ont commencé aussitôt des événements horribles, et l'année 1991." (Poutine, 17 novembre 2011)

И МЫ ТОЖЕ, МЫ ПЕРЕЖИЛИ, ПО СУТИ, РАЗВАЛ ГОСУДАРСТВА: СОВЕТСКИЙ СОЮЗ РАСПАЛСЯ А ЧТО ТАКОЕ СОВЕТСКИЙ СОЮЗ? ЭТО РОССИЯ И ЕСТЬ, ТОЛЬКО НАЗЫВАЛАСЬ ПО-ДРУГОМУ. (ПУТИН, 17.10.2011)

« Nous aussi, de fait, nous avons connu l'effondrement de notre État, l'Union soviétique s'est effondrée. Et c'est quoi, l'Union soviétique ? C'est la Russie, sauf qu'elle s'appelait autrement. » (Poutine, 17 octobre 2011)

Le commentaire, dans la stratégie d'information, malgré son apparence de neutralité a pour objectif de se projeter sur l'époque contemporaine et de souligner implicitement ses avantages. Dans l'exemple suivant, il s'agit de mettre en valeur la liberté de conscience actuelle, en la présentant, à travers la comparaison, comme un des acquis sociaux actuels :

ВЫ ЗНАЕТЕ, У НАС В СТРАНЕ ЕСТЬ ОЧЕНЬ ТЯЖЁЛЫЕ ВОСПОМИНАНИЯ НАЧАЛЬНОГО ПЕРИОДА СОВЕТСКОГО ВРЕМЕНИ, КОГДА ПОСТРАДАЛО ОГРОМНОЕ КОЛИЧЕСТВО СВЯЩЕННИКОВ, ПРИЧЁМ НЕ ТОЛЬКО ПРАВОСЛАВНЫХ СВЯЩЕННИКОВ, НО И МУСУЛЬМАН, И ПРЕДСТАВИТЕЛЕЙ ДРУГИХ РЕЛИГИЙ. (ПУТИН, 06.09.2012)

«Vous savez dans notre pays on a de très mauvais souvenirs de l'époque du début de l'Union soviétique, où un nombre énorme de prêtres ont souffert, et pas seulement des orthodoxes, des musulmans aussi, et des représentants des autres religions. » (Poutine, 6 septembre 2012)

D'ailleurs, cette tactique de commentaire est assez souvent accompagnée de l'accentuation de l'information positive, qui débouche sur la proposition de solutions. La crédibilité du pouvoir en ressort renforcée, car ce panachage de tactiques réduit la distance entre le discours politique et l'action:

КОГДА СТРОИЛИСЬ СОВЕТСКИЕ ЦЕНТРЫ, ОНИ БЫЛИ ПЕРЕДОВЫМИ ВО ВСЕМ. БЫЛА НЕКАЯ ЭКСКЛЮЗИВНОСТЬ. КАЖДЫЙ, КТО ПОПАДАЛ ТУДА, ЗНАЛ, ЧТО ОН ЛУЧШИЙ И НАХОДИТСЯ СРЕДИ ЛУЧШИХ, ОН ДВИГАЕТ СТРАНУ ВПЕРЕД. ВСЕ ЭТИ ПАРАМЕТРЫ НУЖНЫ И СЕГОДНЯ. (СУРКОВ, in ГЛИНКИН, КОСТЕНКО, 15.02.2010)

«Quand ont été construits les centres soviétiques, ils étaient à la pointe sur tous les plans. Exceptionnels, en quelque sorte. Celui qui y était envoyé savait qu'il était le meilleur et qu'il était entouré des meilleurs, qu'il faisait avancer le pays. Tous ces paramètres, on en a besoin aujourd'hui. » (Sourkov, 15 février 2010)

La stratégie d'information et d'interprétation, avec la tactique de proposition de solutions, se distingue par des marqueurs formels, tels que la modalité dans les verbes et des adjectifs de qualification positive. La fonction de ces marqueurs consiste à neutraliser l'attitude négative des interlocuteurs par rapport à la situation actuelle et à renforcer la crédibilité du pouvoir : 
ЧТО БЫ Я ДЕЛАЛ? [...] НУЖНО БЫЛО СВОЕВРЕМЕННО В СОВЕТСКОМ СОЮЗЕ НАЧИНАТЬ ЭКОНОМИЧЕСКИЕ ПРЕОБРАЗОВАНИЯ И РЕФОРМЫ И ЗАКРЕПИТЬ ИХ ДЕМОКРАТИЧЕСКИМИ ПРЕОБРАЗОВАНИЯМИ В СТРАНЕ. (ПУТИН, 15.12.2011)

«Qu'est-ce que j'aurais fait? [...] Il aurait fallu en temps utile commencer les réformes économiques en URSS, et les consolider par une mutation démocratique dans le pays. » (Poutine, 15 décembre 2011)

В 1920-1930-Х ГГ. БЫЛА ЭНЕРГИЯ СТРАХА. БОЛЬШЕВИКИ ХОТЯ БЫ БОЯЛИСЬ, ОНИ ВОЕВАЛИ СО ВСЕМ МИРОМ, ОНИ ОТ СТРАХА НАГРОМОЗДИЛИ СВЕРХДЕРЖАВУ И СОЗДАЛИ ПУСТЬ НЕСОВЕРШЕННУЮ, НО ВНУШИТЕЛЬНУЮ СИСТЕМУ ВООРУЖЕНИЙ, СДЕЛАЛИ РОССИЮ ИНДУСТРИАЛЬНОЙ СТРАНОЙ. ПОСТИНДУСТРИАЛЬНОЕ ОБЩЕСТВО С ПЕРЕПУГУ НЕ ВОЗНИКНЕТ. НУЖНА ПОЗИТИВНАЯ СОЗИДАТЕЛЬНАЯ ЭНЕРГИЯ. (СУРКОВ, in ГЛИНКИН, КОСТЕНКО 15.02.2010)

«Dans les années 1920-30 il y avait l'énergie de la peur. Les Bolcheviks au moins avaient peur, ils étaient en guerre avec le monde entier, et par peur ils ont fait une superpuissance, ils ont créé un système de défense qui n'était pas parfait mais qui impressionnait, ils ont fait de la Russie un pays industrialisé. La société postindustrielle ne naîtra pas par la peur. Il faut une énergie constructive positive.» (Sourkov, 15 février 2010)

Établissant ce parallèle entre l'époque soviétique et la Russie actuelle, le pouvoir se veut pragmatique, en pointant des pratiques négatives, mais aussi positives, du passé, dont certaines pourraient être réactualisées aujourd'hui.

Enfin, vouloir établir la vérité suppose également d'argumenter en recourant à des illustrations tirées d'exemples personnels. Malgré son aspect subjectif, l'illustration semble être une stratégie efficace, dans la mesure où elle rejoint les représentations doxiques. Le propos suivant est d'autant plus crédible qu'il est conforme au vécu collectif. En effet, au moment de la désagrégation de l'URSS, l'assimilation entre l'ancien État et la Russie était très répandue dans la conscience populaire :

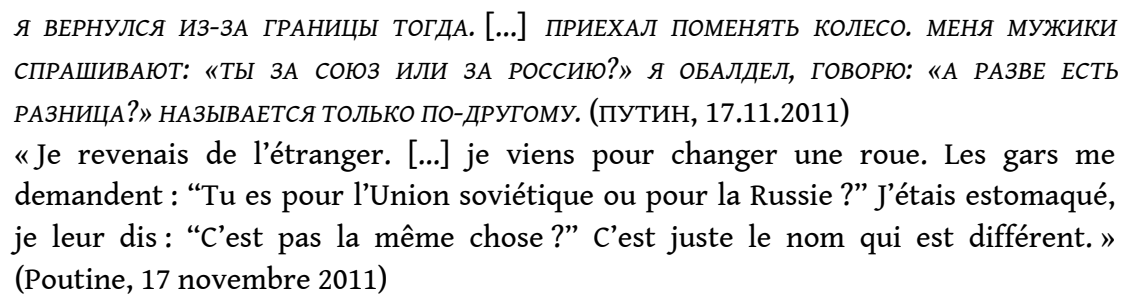

54 Ainsi, les stratégies du discours répondant à l'enjeu de crédibilité se traduisent principalement par des procédés consistant à interpréter ou à commenter les différents phénomènes de la période soviétique et le moment de la désagrégation de l'URSS. Les stratégies d'information sont accompagnées de propositions hypothétiques de solutions. Le pouvoir soit se met en position de neutralité, soit prend position sans sortir du cadre des éléments doxiques, ce qui lui évite de se montrer ouvertement critique ou, au contraire, favorable au passé. Le commentaire de la période soviétique est également employé dans le but de renforcer la crédibilité propre à un pouvoir qui se veut pragmatique et débarrassé des idéologies du passé. Est-ce suffisant pour rester crédible, voire pour capter les interlocuteurs et les séduire? Quelle est la place des soviétismes dans les stratégies liées à l'enjeu de la captation? 


\section{L'enjeu de la captation}

55

l'époque contemporaine, dans la mesure où l'image de Soi est confrontée à l'Autre soviétique, représenté parfois d'une manière exagérément caricaturale. Cette hyperbolisation des défauts de l'Autre fait partie de la stratégie de manipulation. Certes, l'exemple qui suit, celui de l'intervention de Dmitri Medvedev devant les membres du parti du pouvoir "Russie unie", ne semble pas refléter l'intention de manipuler les camarades du parti. Toutefois, elle consiste à dramatiser la situation afin de dynamiser les activités de « Russie unie » à la veille des élections législatives de 2011 : 
60 atégie de dramatisation avec effet théâtral peut s'avérer assez productive grâce au réemploi des métaphores soviétiques comportant un changement de sens ou une inversion de connotation. Ainsi, dans l'exemple suivant, de la métaphore soviétique du " train blindé », qui avait vocation à attribuer une appréciation positive à un phénomène, on ne garde que la forme, pour donner une appréciation négative à l'économie russe :

НО Я ВЕРЮ, ЧТО ЭТО НАДО ДЕЛАТЬ, ПОТОМУ ЧТО СЕГОДНЯ РОССИЙСКАЯ ЭКОНОМИКА ПОХОЖА НА СТАРЫЙ БРОНЕПОЕЗД БЕЗ ЛОКОМОТИВА. НА НЕМ СИДЯТ ЛЮДИ С КОМПЬЮТЕРАМИ И В ГАЛСТУКАХ И ГЛАМУРНЫЕ ДАМЫ, А ЕГО БРОНЯ УЖЕ ПОЧТИ ОСЫПАЛАСЬ И САМ ОН ЗАМЕДЛЯЕТ ХОД. (СУРКОВ, 15.02.2010)

« Mais j'estime qu'il faut le faire, parce qu'aujourd'hui l'économie russe ressemble à un vieux train blindé sans locomotive. Dedans il y a des hommes avec des ordinateurs et en costume cravate, et des dames glamour, mais le blindage est déjà presque complètement parti, et le convoi est en train de s'arrêter. " (Sourkov, 15 février 2010)

61 Les métaphores corporelles, fréquentes dans le discours soviétique, sont également employées dans le cadre de la stratégie de dramatisation, pour atteindre un impact émotionnel. L'exemple qui suit est assez représentatif de l'usage de la métaphore dans la construction de l'image négative de l'Autre actualisé et concret, ici la Grande-Bretagne, contrairement à l'Autre du passé, le PCUS :

[...] В ВЕЛИКОБРИТАНИИ СКРЫВАЮТСЯ ЛЮДИ, У КОТОРЫХ РУКИ ПО ЛОКОТЬ В КРОВИ, КОТОРЫЕ РЕАЛЬНО ВОЕВАЛИ ЗДЕСЬ, НА НАШЕЙ ТЕРРИТОРИИ, С ОРУЖИЕМ В РУКАХ, ЛЮДЕЙ УБИВАЛИ. (ПУТИН, 06.09.2012)

«En Grande-Bretagne se cachent des personnes qui ont les mains dans le sang jusqu'aux coudes, qui ont combattu ici sur notre territoire, les armes à la main, qui ont tué des gens. » (Poutine, 6 septembre 2012)

62 Outre les métaphores, la représentation de l'Autre se fait également par l'exagération avec des moyens lexicaux spécifiques, comme les grossièretés entachées de fortes connotations émotionnelles. La stratégie de dramatisation est dans ce cas proche de celle de la manipulation, car sont présentées d'une manière simpliste et hyperbolisée les règles des rassemblements publics en Grande-Bretagne: «Vot $v$ Londone opredelili mesto. Gde nel'zja, b'jut dubinoj po baške. Nel'zja? Prišel? Poluči, tebja otovarili. » ("À Londres on a dit où c'est permis. Là où on n'a pas le droit, on se prend un coup de matraque sur la tête. C'est interdit ? Et t'es là ? Tiens, prends ça, t'es servi. » Poutine, 30 août 2010).

Le champ lexical des realia soviétiques participe également au développement des stratégies de dramatisation ou de polémique, et ces mots ont pour fonction de produire un effet émotionnel ou de connivence. Outre les exemples de références aux realia soviétiques connotés positivement, d'autres peuvent être utilisés lorsqu'il s'agit de créer un effet négatif. C'est notamment le cas de mots comme lišenec, vrag, agent.

Dans l'exemple suivant, le référent lišenec ${ }^{2}$ n'a pas perdu ses traits caractéristiques d'origine, et l'intention de l'auteur est non seulement de créer un effet de connivence avec les interlocuteurs, mais aussi de produire, sous forme polémique, un effet d'émotion et de contraste entre le passé et l'époque actuelle, entre la réalité soviétique et les fonctionnaires d'aujourd'hui : «Nel'zja prevraščat' gosudarstvennyh ljudej v lišencev kakix-to... » («On ne peut pas transformer des hommes d'État en "sans droits”...» Sourkov, 15 février 2010)

Ainsi, les stratégies du discours participant de l'enjeu de la captation sont déployées davantage au niveau du lexique et des métaphores connotés comme soviétiques, que dans la polémique destinée à formuler une opinion critique du passé. Ces comparaisons et 
analogies avec le passé renforcent d'une manière générale le rôle que jouent les soviétismes dans les stratégies du discours, et qui consiste principalement en la construction de l'image de Soi et de l'Autre et vise l'objectif plus général de l'autoreprésentation.

\section{Conclusion : les soviétismes et l'autoreprésentation}

Le constat que nous pouvons faire après l'étude des soviétismes dans les stratégies du discours du pouvoir russe actuel nous amène d'abord à souligner le rôle prépondérant des références explicites à la période soviétique et à la désagrégation de l'URSS dans les stratégies d'autoreprésentation. En effet, le pouvoir actuel tente de trouver sa légitimité mais aussi sa crédibilité dans le positionnement de Soi par rapport au passé. La stratégie d'autoreprésentation se réalise par des tactiques d'opposition ou de distanciation vis-àvis de l'époque soviétique, pour souligner une autorité soit institutionnelle, soit personnelle du communicant. La tactique d'association, lorsqu'elle est employée dans la mise en valeur de Soi, se projette davantage sur le peuple ou l'État actuel que sur les valeurs ou les réalités soviétiques.

C'est toujours dans un souci de légitimation et d'autoreprésentation que les soviétismes sont réactualisés dans les stratégies du discours sur le plan de l'implicite. L'objectif en est de s'identifier aux interlocuteurs ayant connu l'époque soviétique, c'est-à-dire à la population encore majoritaire aujourd'hui. Il s'agit donc non seulement de construire une image de Soi, mais d'y associer toute cette population.

La réactualisation des soviétismes est moins présente dans les stratégies visant « l'établissement de la vérité ». En effet, l'interprétation de la période soviétique se fait de manière nuancée de sorte à ne pas prendre une position forte pour ou contre le passé. En revanche, les stratégies d'information, malgré leur neutralité apparente, s'inscrivent, elles aussi, dans l'objectif général de se légitimer par la constitution d'une image du régime soviétique qui se veut crédible.

69 L'accentuation de l'image positive de Soi se manifeste également lorsqu'il s'agit de l'enjeu de captation. En effet, en recourant à la stratégie de polémique ou de dramatisation, en grossissant certains défauts du passé, le pouvoir tente de se montrer distant du régime soviétique, tout en évitant sa critique frontale.

De toutes ces stratégies, ressort une intention plus générale qui, nous semble-t-il, vise à trouver un point de départ pour se réconcilier avec le passé, qui pourrait constituer le fondement d'une nouvelle idéologie conservatrice, pouvant trouver l'approbation de l'électorat des couches moyennes de la société russe.

71 Il s'agit principalement des quadragénaires, qui ont gardé en mémoire les dernières années du régime soviétique, et plus particulièrement l'époque de la perestroïka. Cependant, c'est aussi la génération qui a traversé les années turbulentes de l'époque eltsinienne et a fait carrière surtout pendant les années 2000. Par conséquent, la comparaison entre l'époque de Gorbatchev et celle de Eltsine, dans la conscience de cette majorité, n'est pas toujours en faveur de cette dernière, ce qui favorise les sentiments conservateurs de cette partie de la population et leur méfiance par rapport aux valeurs du libéralisme des années 1990.

72 C'est donc à cette partie majoritaire de la population russe, caractérisée par son conservatisme et une mémoire collective positive de l'époque soviétique, que sont 
adressés les soviétismes du discours du pouvoir sous leurs différentes formes, dont nous avons tenté de faire un classement.

Par ailleurs, étant donné l'intérêt du pouvoir russe actuel pour le développement de nouvelles technologies et de l'innovation, nous ne sommes pas étonné de constater dans son discours l'emploi fréquent des anglicismes. Sans avoir fait une estimation quantitative de l'emploi de ces néologismes, nous constatons toutefois la tendance à les utiliser plus souvent que les soviétismes, ce qui s'explique, d'une part, par le rajeunissement actuel de l'électorat et, d'autre part, par l'intérêt de la nouvelle génération des 20 ans, ceux qui sont nés pendant la période post-soviétique, aussi bien pour la politique, avec des mouvements de jeunes partisans de divers partis, que pour les nouvelles technologies.

Pour le moment, le discours du pouvoir tente à la fois de rassurer la majorité conservatrice des Russes par les références au passé soviétique et de se donner une allure moderne, indissociable de son époque, et non pas attardée dans le passé, par un emploi large de néologismes provenant pour la plupart de l'anglais.

\section{BIBLIOGRAPHIE}

БУДАЕВ Э.В. \& ЧУДИНОВ А.П. (2009), ЛИНГВИСТИЧЕСКАЯ СОВЕТОЛОГИЯ, Ekaterinbourg : УРГПУ.

ВЕРЕТЕНКИНА Л.Ю. (2001), « СТРАТЕГИЯ, ТАКТИКА И ПРИЕМЫ МАНИПУЛИРОВАНИЯ », ЛИНКВОКУЛЬТУРОЛОГИЧЕСКИЕ ПРОБЛЕМЫ ТОЛЕРАНТНОСТИ: ТЕЗИСЫ ДОКЛАДОВ МКЖДУНАРОДНОЙ НАУЧНОЙ КОНФЕРЕНЦИИ, Ekaterinbourg : ИЗДАТЕЛЬСТВО УРАЛЬСКОГО УНИВЕРСИТЕТА.

CHABRol Claude (1990), « Réguler la construction de l'identité du sujet du discours »,

A. Berrendonner et H. Parret (dir.), L'interaction communicative, Berne : Peter Lang.

Charaudeau Patrick (1994a), « Le discours publicitaire, genre discursif », Mscope, 8, Versailles : CRDP.

CHARAUDEAU Patrick (1994b), « Le contrat de communication médiatique », Le français dans le monde, numéro spécial, « Médias, faits et effets », 8-19.

Charaudeau Patrick (1998a), « La télévision peut-elle expliquer ? », P. Bourdon et F. Jost (dir.), Penser la télévision, Paris : Nathan.

CHARAUDEAU Patrick (1998b), « L'argumentation n'est peut-être pas ce qu'on croit », Le français d'aujourd'hui, 123.

Charaudeau Patrick (2000), « Une problématique discursive de l'émotion. À propos des effets de pathémisation à la télévision », C. Plantin, M. Douty et V. Traverso (dir.), Les émotions dans les interactions, Lyon : Presses universitaires de Lyon.

ESPERET Éric (1990), « Apprendre à produire du langage : construction des représentations et processus cognitifs ", Le français dans le monde, numéro spécial dirigé par D. Gaonac'h, « Acquisition et utilisation d'une langue étrangère. L'approche cognitive », 8-15. 
ИССЕРС О.С. (1999), КОММУНИКАТИВНЫЕ СТРАТЕГИИ И ТАКИТИКИ РУССКОЙ РЕЧИ, omsk : ИЗДАТЕЛЬСТВО ОГУ.

КАТЦЕР Ю.М. \& КУНИНА А.В. (1964), ПИСЬМЕННЫЙ ПЕРЕВОД С РУССКОГО ЯЗЫКА НА АНГЛИЙСКИЙ, MOscou : ВЫСШАЯ ШКОЛА.

МАКАРОВ М.Л. (2003), ОСНОВЫ ТЕОРИИ ДИСКУРСА, MOscou : ИТДГК ГНОЗИС.

MAzon André (1920), Lexique de la guerre et de la révolution en Russie, Paris : Champion.

ТРУФАНОВА И.О. (2001), « О РАЗГРАНИЧЕНИИ ПОНЯТИЙ: РЕЧЕВОЙ АКТ, РЕЧЕВОЙ ЖАНР, РЕЧЕВАЯ

СТРАТЕГИЯ, РЕЧЕВАЯ ТАКТИКА », ФИЛОЛОГИЧЕСКИЕ НАУКИ, 3.

VON NEUMANN John \& MORGENSTERN Oskar (1964), Theory of Games and Economic Behavior, New York : J. Wiley \& Sons.

ЧЕРНОВ Г.В. (1958), ВОПРОСЫ ПЕРЕВОДА РУССКОЙ БЕЗЭКВИВАЛЕНТНОЙ ЛЕКСИКИ НА АНГЛИЙСКИЙ ЯЗЫК (НА МАТЕРИАЛЕ ПЕРЕВОДОВ СОВЕТСКОЙ ПУБЛИЦИСТИКИ), АВТОРЕФЕРАТ ДИССЕРТАЦИИ К. Ф. Н., Moscou.

\section{Corpus d'exemples}

ГЛИНКИН М. \& КОСТЕНКО Н., «ЧУДО ВОЗМОЖНО, - ВЛАДИСЛАВ СУРКОВ, ПЕРВЫЙ ЗАМРУКОВОДИТЕЛЯ АДМИНИСТРАЦИИ ПРЕЗИДЕНТА, ЗАМПРЕДСЕДАТЕЛЯ КОМИССИИ ПО МОДЕРНИЗАЦИИ», ВЕДОМОСТИ, 15 février 2010.

« ИНТЕРВЬЮ ПРЕЗИДЕНТА РОССИИ ДМИТРИЯ МЕДВЕДЕВА ТЕЛКАНАЛАМ ПЕРВЫЙ, РОССИЯ И НТВ ॥, 30 septembre 2011, <www.kremlin.ru/transcripts/12880> (12 novembre 2014).

« ИНТЕРВЬЮ ПРЕДСЕДАТЕЛЯ ПРАВИТЕЛЬСТВА РОССИЙСКОЙ ФЕЛЕРАЦИИ В.В. ПУТИНА 》, 17 octobre 2011, <http://archive.premier.gov.ru/events/news/16755/> (12 novembre 2014).

« ИНТЕРВЬЮ ТЕЛЕКАНАЛУ Russia Today », 6 septembre 2012, <www.kremlin.ru/ transcripts/16393> (12 novembre 2014).

КОЛЕСНИКОВ А., « ВЛАДИМИР ПУТИН: ДАЮ ВАМ ЧЕСТНОЕ ПАРТИЙНОЕ СЛОВО », КОММЕРСАНТЪ, 30 août 2010.

МЕДВЕДЕВ Д.А., « ВСТРЕЧА ДМИТРИЯ МЕДВЕДЕВА СО СТОРОННИКАМИ », 15 octobre 2011, <http:// kremlin.ru/transcripts/13065> (12 novembre 2014).

« ПРЕСС-КОНФЕРЕНЦИЯ ПО ИТОГАМ ФОРУМА АТЭС », 9 septembre 2012, <http://kremlin.ru/ transcripts/16432> (12 novembre 2014).

« РАЗГОВОР С ВЛАДИМИРОМ ПУТИНЫМ. ПРОДОЛЖЕНИЕ », 16 décembre 2010, < http://2010.moskva-putinu.ru/> (12 novembre 2014).

СУББОТИНА Е., « ГЕНЕРАЛЬНУЮ УБОРКУ АРКТИКИ ЗАВЕРШАТ В 2020 ГОДУ », РОССИЙСКАЯ ГАЗЕТА, 16 octobre 2012.

« СТЕНОГРАММА ВСТРЕЧИ ПРЕЗИДЕНТА И ПРЕМЬЕРА С ПЕНСИОНЕРАМИ И ВЕТЕРАНАМИ », РОССИЙСКАЯ ГАЗЕТА, 17 novembre 2011.

« СТЕНОГРАММА ПРОГРАММЫ РАЗГОВОР С ВЛАДИМИРОМ ПУТИНЫМ. ПРОДОЛЖЕНИЕ », РОССИЙСКАЯ ГАЗЕТА, 15 décembre 2011. 


\section{NOTES}

1. Nous avons fait une traduction littérale du mot russe uvažaemyj, employé par D. Medvedev dans une phrase affirmative qui n'est pas adressée directement aux personnes mentionnées. En réalité, ce mot associé à l'autre « camarade » est une expression d'adresse de l'époque soviétique. Afin de créer l'effet comique Medvedev place cette expression dans un propos dont l'objectif ne consiste pas à s'adresser aux personnes concernées mais à les évoquer, ce qui produit un décalage entre l'attente de l'interlocuteur et le résultat de l'énoncé.

2. Une catégorie de citoyens soviétiques à l'époque stalinienne privée de droits civiques sur la base de critères de classe.

\section{RÉSUMÉS}

Dans le présent article nous tentons d'évaluer le rôle et les fonctions des soviétismes dans les stratégies de discours du pouvoir russe contemporain. En effet, le discours politique russe en général, et le discours du pouvoir en particulier, ont subi de multiples modifications, allant des transformations lexicales jusqu'aux choix argumentatifs multiples s'inscrivant dans les nouvelles stratégies de communication. Malgré sa volonté de moderniser le discours politique, le pouvoir russe contemporain s'appuie pourtant d'une manière récurrente, dans ses stratégies de communication, sur des éléments de vocabulaire, mais aussi sur certaines représentations sociétales et culturelles soviétiques, afin d'atteindre certains objectifs de communication et, particulièrement, ceux de l'autoreprésentation, qui s'inscrivent plus globalement dans une politique d'autolégitimation.

This study analyses different modes of re-actualization of sovietisms in contemporary Russian political discourse. Based on a corpus including interviews and public speeches by Russian political leaders this paper is focused upon different ways of introducing sovietisms in the political discourse (implicit references, metaphors or explicit intentional use) on one hand, and functions of sovietisms in the building of communicative strategies of Russian political power on the other. This paper highlights the correlation between transformed sovietisms in the contemporary political discourse and the target public represented by the middle Russian electorate.

\section{INDEX}

Mots-clés : discours politique, pouvoir russe contemporain, soviétisme, stratégie de discours, communication politique

Keywords : political discourse, Russian political authorities, sovietism, strategy of discourse, communication 


\section{AUTEUR}

\section{VALÉRY KOSSOV}

Université Grenoble Alpes, ILCEA4 (CESC) 